\title{
SHM systems supporting extension of the structures' service life
}

\author{
Wolfgang R. Habel
}

(C) Springer-Verlag Berlin Heidelberg 2013

Many engineering structures are decades old and need to be refurbished. Some of them may even need to be replaced. In most cases, replacement is not possible in the short-term because of the high investment expenses even if the functionality of the structure is definitely limited. It is obvious that a growing number of buildings, ground structures and bridges are reaching or have exceeded their expected life cycle, and that this requires more attention, presently and much more in the future. If you have to solve such problems, there are several avenues open: one is to reduce service loads, which could lead to problems in transport logistics. An alternative way is permanently monitor the structure's behaviour with the objective to recognize significant risks early or extend the structure's design service life. Given the high number of damaged or aged structures, a method of service life estimation and extension is an area of increasing importance within civil and structural engineering profession.

Aware of this trend to ensure structural health and handle upcoming problems with aged structures more efficiently, ISHMII-The International Society for Structural Health Monitoring of Intelligent Infrastructure (http://www.ishmii.org), decided to present a special issue of its Journal of Civil Structural Health Monitoring (JCSHM) that discusses the topic "SHM systems supporting extension of the structures' service life". The JCSHM Editorial Board invited international experts including civil engineers, academics, designers, construction companies, product and monitoring system managers as well as responsible persons from government agencies

W. R. Habel $(\bowtie)$

Federal Institute for Materials Research and Testing (BAM), Berlin, Germany

e-mail: wolfgang.habel@bam.de and road, highway and waterways authorities to submit research findings. Outstanding authors were also asked to publish recent methodologies and new technical solutions for the management of critical infrastructure, all based on monitoring where structural health monitoring includes assessment methodologies that enable life extension of different type of structures, e.g. aged or damaged railway bridges, ancient structures or large concrete bridges.

We are proud to present this special issue to you with its selected and peer-reviewed contributions. The articles range from the use of a probabilistic method to allow a bridge owner to weigh the risk of maintaining a bridge past its design service life, and a special 'Relaxation Monte Carlo Filter' algorithm for better identification of dynamic parameters of structural systems and a time-frequency analysis tool to reveal a change of the characteristics in vibration signal, to new detection methods for the assessment of the structure's condition or to evaluate important structure components such as pre-stressed elements, bridge cables or ropes. We have not forgotten important practicerelevant details such as how to place sensors or what to do if sensor data are inhomogeneous; these are also considered in this issue. Moreover, an extended monitoring system to observe the behaviour of the famous very old and still used Roman Arena in Verona, Italy and to estimate its response in the case of exceptional events, like an earthquake, is described.

As practitioners, we all know that SHM hardware solutions suggested to extend a structure's service life will have to be measured against the economic case. So, we also offer papers that address important aspects on how to ensure an economic return when SHM systems are applied to new or aged structures in this special issue. The spectrum of papers that were submitted and those selected was very broad; most dealt with advanced monitoring 
strategies. Of course, more interesting papers were submitted than we could publish in this issue. In order to make these contributions available to our readers, our broad expert and user community, some of these papers will be published in the next regular issues of the JCSHM.

As guest editor, I believe that this special issue and the up-coming issues of this still young Journal show its attractiveness and make it more and more exiting to a wide readership. If you, as a loyal reader or maybe as a new JCSHM reader, want to be kept up-to-date on the highlights in SHM and related techniques, you are invited to join ISHMII-the global society-and take advantage of discussions at ISHMII workshop and conferences, and in expert groups, to bring your competence in and draw added value for effective performance and ageing management as well as economic decisions. Knowledge exchange and outstanding expertise are the keys to a proper decision on whether a structure should be repaired or replaced or whether it could remain in service without any danger while being monitored.

Enjoy reading this special issue.

\section{Wolfgang R. Habel}

Guest Editor 